\title{
Regional myocardial contractile reserve assessed by strain echocardiography and the response to cardiac resynchronization therapy
}

\author{
Peter Mitro, Pavol Murin, Gabriel Valocik, Milos Simurda \\ Cardiology Clinic, Safarik University and VUSCH Kosice, Slovak Republic
}

\begin{abstract}
Background: Overall response rate to cardiac resynchronization therapy (CRT) is still not optimal. The aim of the study was to evaluate the influence of the regional myocardial contractile reserve during dobutamine infusion in the area of left ventricular (LV) electrode on the response rate and reverse remodeling $L V$ in patients receiving $C R T$.

Methods: Biventricular pacemaker was implanted in 41 consecutive patients (33 men, mean age $62 \pm 10$ years) with LV ejection fraction $(L V E F) \leq 35 \%$, New York Heart Association class III and $Q R S$ duration $\geq 120$ ms. Myocardial contractile reserve was assessed by LV strain during dobutamine infusion (20 $\mathrm{gg} / \mathrm{kg} / \mathrm{min})$ using speckle tracking echocardiography. Patients were classified as responders if an increase in LVEF $\geq 5 \%$ or decrease in end-systolic volume $\geq 15 \%$ was observed after 6 months of CRT.
\end{abstract}

Results: Twenty-four patients were responders and 17 were non-responders. During dobutamine infusion at a rate of $20 \mu \mathrm{g} / \mathrm{kg} / \mathrm{min}$, responders showed significant increase in regional deformation ( $\Delta$ strain) when compared to non-responders $(2.14 \pm 2.9$ vs. $-0.94 \pm 1.74, p=0.042)$. Patients with increased deformation in the LV lead area during dobutamine stimulation were more likely to be responders to CRT compared to patients without increased deformation in this area (81\% vs. $20 \%, p=0.0002)$. They exhibited significant increase in LVEF $(8.8 \% \pm 10.3 \%$ vs. $0.3 \% \pm 6.4 \%, p=0.01)$. LV electrode localization in viable myocardium was a good predictor of response to CRT (AUC 0.852, $p<0.0001$ ).

Conclusions: Regional contractile reserve assessed by strain rate echocardiography during dobutamine infusion predicts the response to CRT. (Cardiol J 2014; 21, 5: 524-531)

Key words: myocardial contractility, cardiac resynchronization therapy, myocardial strain, echocardiography, myocardial viability

\section{Introduction}

Cardiac resynchronization therapy (CRT) is an important therapeutic option in drug-refractory heart failure (HF) of ischemic and non-ischemic etiology. CRT reduces symptoms of $\mathrm{HF}$ and re- duces morbidity and mortality. In comparison to pharmacological therapy, CRT lowers the hospitalization for $\mathrm{HF}$ by $52 \%$ and leads to relative risk reduction in cardiac death of $36-40 \%$ [1]. However, the overall response rate to CRT is still not optimal. About one third of patients do not respond

Address for correspondence: Prof. Peter Mitro, MD, PhD, Cardiology Clinic, Safarik University and VUSCH, Ondavska 8, Košice 040 01, Slovak Republic, tel/fax: 0042 1557891315, e-mail: peter.mitro@upjs.sk

Received: 15.10.2013 Accepted: 17.12.2013 
to CRT. Majority of attempts to solve the issue of non-responding patient population is focused on the improvement of patient selection and optimization of device programming after implantation [2]. Lead positioning is another factor contributing to the therapeutic effect of CRT in the individual patient. The optimal site for the positioning of the left ventricular (LV) lead is lateral or posterolateral vein. Anterior location of LV lead is controversial, sub-study from MADIT-CRT trial showed that only apical, not anterior location predicted lower response to CRT [3]. Similarly, analysis of lead location in the REVERSE trial concluded that more favorable outcome of CRT was observed in patients with lateral positioning of LV lead away from the apex. Position of right ventricular (RV) lead was indifferent [4].

Suboptimal LV lead location was reported in $21 \%$ of patients with lack of CRT response [5].

Factors that influence LV lead position are usually periprocedual (adequate pacing threshold and sensing, lead stability and avoidance of diaphragmatic stimulation) [2]. Besides these conventional factors, knowledge of myocardial viability in the area of LV lead may help further optimize the LV lead location and response to CRT.

The aim of the study was to evaluate the influence of the myocardial contractile reserve during dobutamine infusion (as a measure of myocardial viability) in the area of LV electrode on the response rate and reverse remodeling of $\mathrm{LV}$ in patients receiving CRT.

\section{Methods}

The study prospectively enrolled 41 consecutive patients with $L V$ dysfunction, $L V$ ejection fraction (LVEF) $<35 \%$ and New York Heart Association (NYHA) class III with a QRS duration $\geq 120 \mathrm{~ms}$. Patients with recent acute myocardial infarction (MI) ( $<3$ months), acute HF, myocardial revascularization $<6$ months before and with previously implanted pacemaker were excluded from the study.

Ischemic etiology of HF was defined as a history of MI and/or evidence of significant stenosis $(\geq 70 \%)$ on coronary angiography. Coronary artery disease was present in 27 (51\%) patients (Table 1 ). Non-ischemic etiology was defined as absence of significant stenosis on coronary angiography and/or absence of prior MI.

Biventricular pacemaker was implanted in all patients. Electrodes were placed by transvenous way (left subclavian vein). The atrial lead was placed in the right atrium. The RV lead was placed
Table 1. Significant comorbidities and clinical parameters in patients receiving cardiac resynchronization therapy (CRT) $(n=41)$.

\begin{tabular}{lc}
\hline Mean age [years] & $61.9 \pm 10.9$ \\
Men & 33 \\
Women & 9 \\
Mean LVEF [\%] & $26.3 \pm 7.1$ \\
Mean QRS width [ms] & $152.1 \pm 22.3$ \\
LVEDD [mm] & $6.4 \pm 0.8$ \\
LVESD [mm] & $5.6 \pm 0.9$ \\
LVEDV [mL] & $234.1 \pm 71.7$ \\
LVESV [mL] & $174.1 \pm 59.8$ \\
Mean BMI [kg/m $\left.{ }^{2}\right]$ & $25.94 \pm 4.4$ \\
Mean plasma creatinine $[\mu \mathrm{mol} / \mathrm{L}]$ & $116 \pm 43$ \\
Mean hemoglobin $[\mathrm{g} / \mathrm{L}]$ & $13.7 \pm 1.6$ \\
Coronary artery disease & $25(60 \%)$ \\
Diabetes mellitus & $24(57 \%)$ \\
Arterial hypertension & $33(79 \%)$ \\
Atrial fibrillation & $7(16 \%)$ \\
\hline
\end{tabular}

LVEF - left ventricular ejection fraction; LVEDD — left ventricular end-diastolic diameter; LVESD - left ventricular end-systolic diameter; LVEDV — left ventricular end-diastolic volume; LVESV — left ventricular end-systolic diameter; BMI — body mass index

on the septal wall of the RV. The LV lead was positioned into a branch of a coronary sinus.

All patients gave informed consent to perform dobutamine stress echocardiography as part of a study.

\section{Dobutamine stress echocardiography}

Before the implantation standard echocardiographic examination was performed in all patients (Siemens SC 2000, Mountain View, USA). LVEF using a biplane Simpson technique, LV end-systolic and end-diastolic diameter (LVESD, LVEDD), LV end-systolic and end-diastolic volume (LVESV, LVEDV) were recorded. In addition, dobutamine stress echocardiography was carried out.

Dobutamine was diluted in $400 \mathrm{~mL} 0.9 \%$ saline infusion and infused into antecubital vein in the incremental dosage. The infusion rate was gradually increased starting from $5 \mu \mathrm{g} / \mathrm{kg} / \mathrm{min}$ up to $20 \mu \mathrm{g} / \mathrm{kg} / \mathrm{min}$ over $20 \mathrm{~min}$. Baseline standard two-dimensional echocardiographic projections were obtained and stored in a digital cine-loop format for the off-line analysis.

\section{Regional myocardial contractility assessment}

Regional myocardial contractility was assessed in the segment of LV where the stimulation electrode was located. 


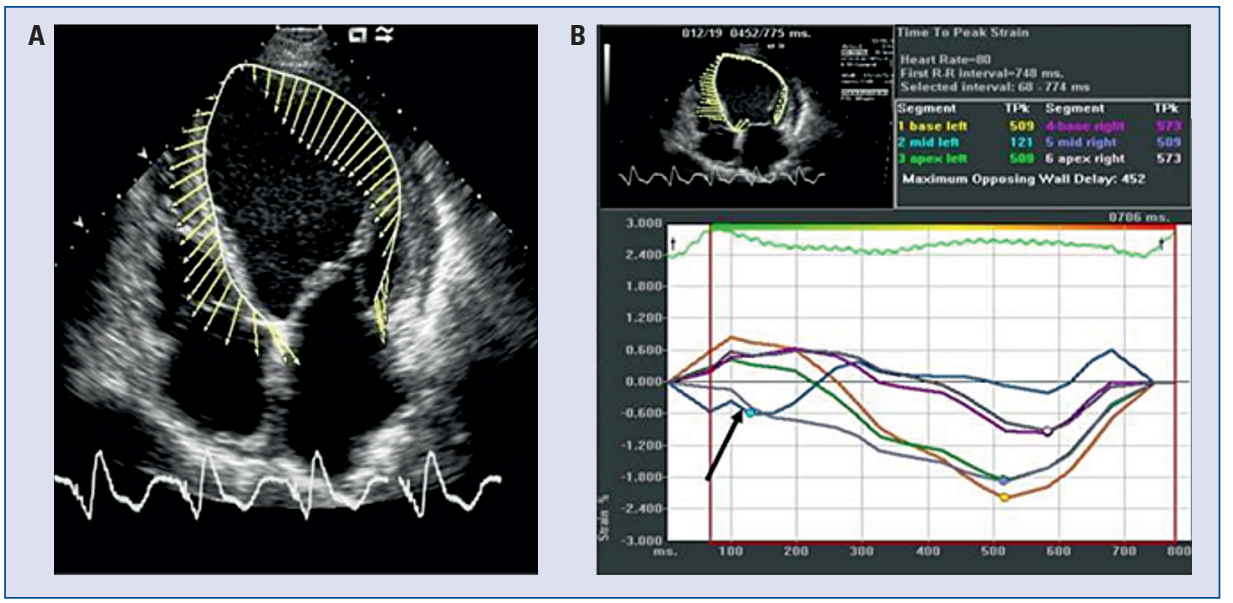

Figure 1. Strain rate evaluation in the apical 4-chamber projection; A. Endocardium is manually traced in end-diastole in apical 4-chamber view. Vectors of myocardial strain are shown by small arrows. Arrows show direction and magnitude of myocardial segment deformations; B. Myocardial strain curves in 6 different segments of left ventricle. Maximal extent of systolic deformation and the time to maximal deformation is detectable in each segment. Arrow points to the segment with the earliest (and the smallest) nadir of systolic deformation.

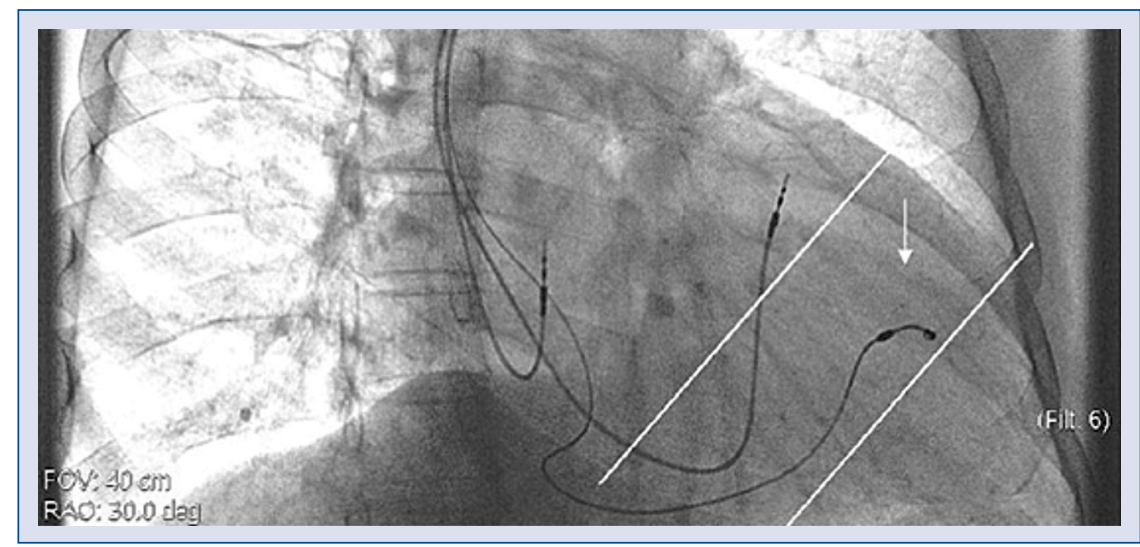

Figure 2. Position of the electrode in RAO 30 projection. Arrow shows the tip of the left ventricular electrode in the middle part of the left ventricle.

Contractility assessment was based on changes in LV strain during dobutamine infusion $(20 \mu \mathrm{g} /$ $/ \mathrm{kg} / \mathrm{min}$ ). Peak systolic radial strain was analyzed by speckle tracking echocardiography.

Viable myocardial segment was defined by an increase of the strain (deformation) during dobutamine infusion. If no change or decrease in deformation of the myocardium was observed, myocardial segment was considered non-viable (Fig. 1).

\section{Position of the left-ventricular electrode}

Chest X-ray was carried out in all 41 patients on the second day following the pacemaker implan- tation in 3 standard projections: anterior-posterior projection (AP), right-anterior oblique projection in 30 degrees (RAO 30) and left-anterior oblique projection in 60 degrees (LAO 60). Anatomic location of the stimulation electrode in the LV was assessed by 2 independent physicians. RAO 30 projection was used to determine the position of the electrode in the frontal plane (Fig. 2). The position of the electrode in the frontal plane was classified as basal-, mid-, and apical-LV position.

In LAO 60 projection, the location of the electrode in the frontal plane was determined using a scheme consisting of 17 segments (Figs. 3, 4). 


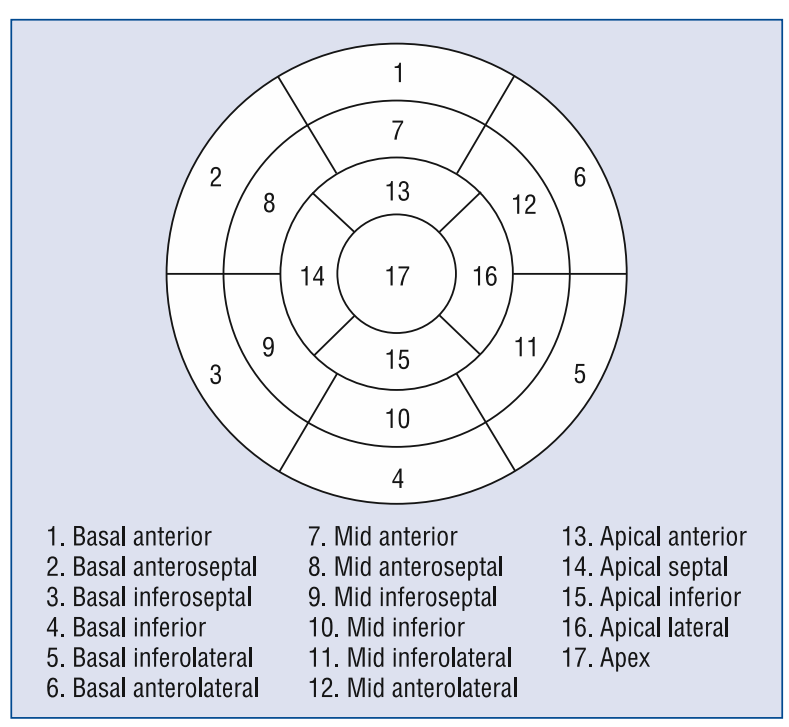

Figure 3. Segments of the left ventricle.

\section{Follow-up}

Patients were followed for 6 months. Follow-up included echocardiographic determination of the LVEF, LVESD, LVEDD, LVESV, LVEDV, as well as clinical assessment of the patient. Patients were classified as responders if they were alive, and an increase in LVEF $\geq 5 \%$ or decrease in end-systolic volume $(\mathrm{ESV}) \geq 15 \%$ was observed at the follow-up. Other patients and patients who died during the follow-up period were classified as non-responders.

\section{Statistical analysis}

Continuous variables are expressed as mean \pm \pm standard deviation. Categorical variables are expressed in frequencies and percentages. Data distribution was tested by Shapiro-Wilk test.
Unpaired Student's t-test was used to test differences between responders and non-responders in parametric continuous variables and $\chi^{2}$ test/ /Fischer's exact test in normally distributed categorical parameters. Mann-Whitney test was used for non-parametric continuous data. Receiver operating characteristics (ROC) analysis was used to assess the usefulness of the myocardial viability in area of the LV electrode in the prediction of CRT response. $\mathrm{P}$ value $<0.05$ was considered statistically significant.

\section{Results}

\section{Responders and non-responders}

Six months after pacemaker implantation $24(58 \%)$ patients were classified as responders and $17(42 \%)$ patients as non-responders based on echocardiographic criteria, as mentioned above. Five patients from the non-responders group died because of a cardiovascular reason. One patient died because of the oncological disease and was excluded from the study. Two patients were lost during the follow-up.

There were no significant differences between group of responders and non-responders in clinical characteristics, laboratory parameters, and pharmacological treatment (Table 2). A non-significant trend to higher level of creatinine and increased frequency of atrial fibrillation was observed in the non-responders group. In the responders LVEF significantly increased after 6 months of follow-up from $24.9 \pm 7 \%$ to $36.5 \pm 7.4 \%(\mathrm{p}<0.0001)$ and ESV decreased from $178 \pm 54 \mathrm{~mL}$ to $129.8 \pm 43 \mathrm{~mL}$ $(\mathrm{p}<0.0001)$.

In the non-responder group, LVEF did not change significantly during the follow-up period

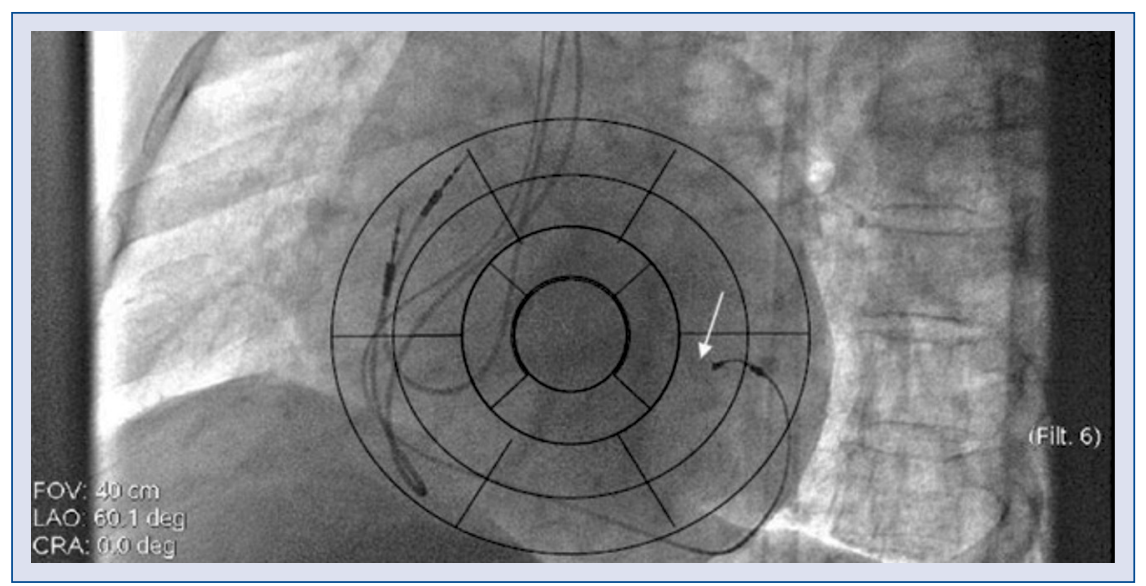

Figure 4. Position of the electrode in LAO 60 projection in the same patient as in Figure 2. Arrow shows the tip of the electrode in inferolateral segment of the left ventricle. 
Table 2. Clinical characteristics in responders and non-responders. All statistical significance: $p=N S$ (non-significant).

\begin{tabular}{lcc}
\hline & Responders $(\mathbf{n}=\mathbf{2 4 )}$ & Non-responders $(\mathbf{n}=\mathbf{1 7})$ \\
\hline Mean age [years] & $62.3 \pm 11.3$ & $61.4 \pm 2.7$ \\
Men & $17(81 \%)$ & $15(79 \%)$ \\
Plasma creatinine $[\mu \mathrm{mol} / \mathrm{L}]$ & $107 \pm 6$ & $123 \pm 16$ \\
Hemoglobin $[\mathrm{g} / \mathrm{L}]$ & $14.1 \pm 1.8$ & $13.3 \pm 1.4$ \\
Body mass index $\left[\mathrm{kg} / \mathrm{m}^{2}\right]$ & $25.1 \pm 3.1$ & $26.3 \pm 5.2$ \\
Coronary artery disease & $12(50 \%)$ & $13(68 \%)$ \\
Diabetes mellitus & $14(64 \%)$ & $10(53 \%)$ \\
Arterial hypertension & $16(73 \%)$ & $17(89 \%)$ \\
Beta-blocker & $20(91 \%)$ & $17(89 \%)$ \\
Angiotensin converting enzyme inhibitor or sartan & $19(86 \%)$ & $13(68 \%)$ \\
Furosemide & $19(86 \%)$ & $16(84 \%)$ \\
\hline
\end{tabular}

$(28.2 \pm 7 \%$ vs. $24.7 \pm 5 \%$, p > 0.1) (Fig. 5). Similarly, ESV did not decrease in the non-responder group $(168.6 \pm 65 \mathrm{~mL}$ vs. $170.2 \pm 51 \mathrm{~mL}, \mathrm{p}>0.1)$.

In the responder group, clinical improvement was also observed, expressed by decreased NYHA class, whereas NYHA class did not change in the non-responder group $(-0.77 \pm 0.6$ vs. $0.14 \pm 0.6$, $\mathrm{p}=0.021)$ (Table 3).

Regional myocardial viability in the

left-ventricular electrode area

Localization of LV electrode in the group of 41 patients is shown in Table 4 . The LV electrode was most frequently located in mid-inferolateral (one third of patients), mid-anterior and mid-anterolateral segments of the LV.

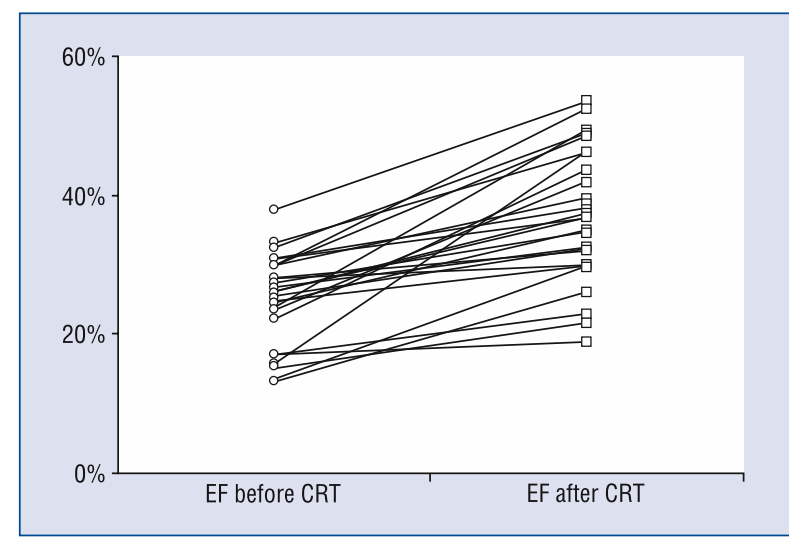

Figure 5. Individual change in ejection fraction (EF) in patients who respond to cardiac resynchronization therapy (CRT).

Table 3. Echocardiographic parameters and New York Heart Association (NYHA) classification before cardiac resynchronization therapy (CRT) and 6 months after CRT implantation in responders and non-responders.

\begin{tabular}{lccc}
\hline & Responders & Non-responders & P \\
\hline Before CRT & & & $\mathrm{NS}$ \\
EF [\%] & $24.9 \pm 7.0$ & $28.2 \pm 7.1$ & $\mathrm{NS}$ \\
LVESV [mL] & $178.0 \pm 54.8$ & $168.6 \pm 65.8$ & $\mathrm{NS}$ \\
LVEDV [mL] & $234.9 \pm 62.5$ & $233.0 \pm 82.9$ & $\mathrm{NS}$ \\
LVESD [mm] & $5.7 \pm 0.8$ & $5.4 \pm 1.1$ & $\mathrm{NS}$ \\
LVEDD [mm] & $6.5 \pm 0.5$ & $6.4 \pm 0.9$ & \\
After 6-month follow-up & & & $<0.0001^{*}$ \\
$\Delta$ EF [\%] & $11.6 \pm 8.1$ & $-3.5 \pm 5.3$ & $<0.0001^{*}$ \\
$\Delta$ LVESV [mL] & $-48.2 \pm 42.4$ & $1.6 \pm 22.0$ & $0.0446^{*}$ \\
$\Delta$ LVEDV [mL] & $-32.8 \pm 45.7$ & $-5.9 \pm 38.1$ & $\mathrm{NS}$ \\
$\Delta$ LVESD $[\mathrm{mm}]$ & $-0.1 \pm 0.8$ & $0.4 \pm 0.7$ & $\mathrm{NS}$ \\
$\Delta$ LVEDD $[\mathrm{mm}]$ & $-0.1 \pm 0.7$ & $0.1 \pm 0.7$ & $0.021^{*}$ \\
$\Delta$ NYHA & $-0.77 \pm 0.6$ & $0.14 \pm 0.6$ & \\
\hline
\end{tabular}

*p < 0.05; EF - ejection fraction; LV — left ventricular; EDV — end-diastolic volume ESV — end-systolic volume; EDD — end-diastolic diameter; ESD - end-systolic diameter 
Table 4. Position of the left-ventricular electrode tip.

\begin{tabular}{lc}
\hline $\begin{array}{l}\text { Left-ventricular } \\
\text { electrode location }\end{array}$ & $\begin{array}{c}\text { Patient } \\
\text { number (\%) }\end{array}$ \\
\hline Basal anterior segment & $4(10 \%)$ \\
Mid anterior segment & $7(17 \%)$ \\
Basal anterolateral segment & $5(12 \%)$ \\
Mid anterolateral segment & $6(15 \%)$ \\
Apical lateral segment & $2(5 \%)$ \\
Basal inferolateral segment & $1(2 \%)$ \\
Mid inferolateral segment & $14(34 \%)$ \\
Apical inferior segment & $2(5 \%)$ \\
\hline
\end{tabular}

Regional myocardial deformation in the area of the LV electrode tip was assessed by the strain analysis (Fig. 1). The myocardial deformation did not differ between responders and non-responders before dobutamine infusion ( $\mathrm{p}=0.0623$ ).

During dobutamine infusion at a rate of $20 \mu \mathrm{g} /$ $/ \mathrm{kg} / \mathrm{min}$, responders showed significant increase in the regional deformation ( $\Delta$ strain) when compared to non-responders $(2.14 \pm 2.9 \%$ vs. $-0.94 \pm 1.74 \%$, $\mathrm{p}=0.042$ ).

Patients with viable myocardium (i.e. with increased deformation during dobutamine stimulation) in the area of LV electrode were more likely to be responders to CRT when compared to patients with LV electrode in non-viable segment of the LV ( $81 \%$ vs. $20 \%, \mathrm{p}=0.0002$ ). They exhibited significant increase in LVEF and a trend (non-significant) to decreased LVESV (Table 5). ROC analysis showed that LV electrode localization in viable segment of the $\mathrm{LV}$ is a good predictor of CRT response (area under curve [AUC] 0.852 , $\mathrm{p}<0.0001$ ) (Fig. 6).

\section{Discussion}

Viable myocardium is characterized by increased contractility in the setting of decreased metabolic demand and/or improved perfusion. The outcome of the CRT depends on myocardial

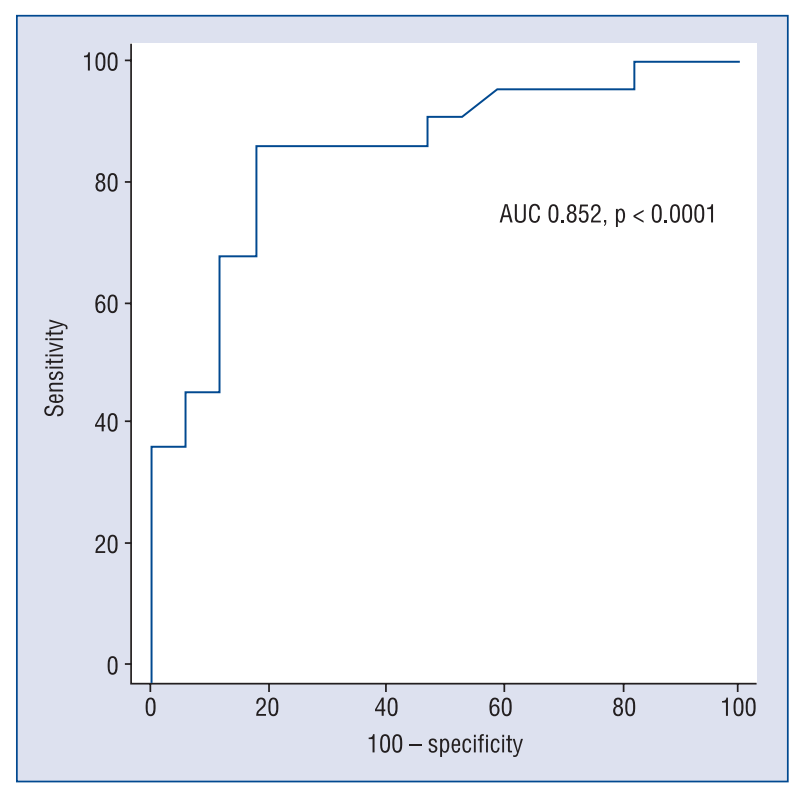

Figure 6. Myocardial viability in area of the left ventricular electrode as a predictor of cardiac resynchronization therapy response; AUC - area under curve.

viability. Interventricular and intraventricular resynchronization of the heart decreases intramural stress and LV end-diastolic pressure and increases coronary perfusion. In the presence of viable myocardium, improved coronary perfusion augments the myocardial contractility and cardiac output $[6,7]$.

Myocardial viability can be measured as a global contractile reserve during dobutamine infusion. An increase of 7.5\% in LVEF during low dose dobutamine infusion was reported as a predictor of the LV reverse remodeling and response to CRT [8].

Viability of the area directly under the LV stimulation electrode is probably another important factor influencing the response to CRT. Implantation of the LV lead into the area of myocardial scar was suggested as one of the potential mechanisms of patients' non-responsiveness to CRT. Cardiac pacing within the area of myocardial scar leads to

Table 5. Influence of myocardial viability in the left ventricular (LV) electrode area on response to cardiac resynchronization therapy.

\begin{tabular}{lccc}
\hline & $\begin{array}{c}\text { Patients with viable LV } \\
\text { electrode area }\end{array}$ & $\begin{array}{c}\text { Patients with non-viable LV } \\
\text { electrode area }\end{array}$ & P \\
\hline$\Delta$ Deformation (strain) [\%] & $2.7 \pm 2.6$ & $-1.7 \pm 1.3$ & 0.0001 \\
Responders/non-responders & $21 / 5$ & $3 / 12$ & 0.0002 \\
$\Delta$ EF after 6 months [\%] & $8.8 \pm 10.3$ & $0.3 \pm 6.4$ & 0.0141 \\
$\Delta$ LVESV after 6 months [mL] & $-35.7 \pm 42.9$ & $-13.5 \pm 41.4$ & NS \\
\hline
\end{tabular}

LVESV — left ventricular end-systolic volume; EF — ejection fraction 
prolonged and fragmented QRS complex and to electrical dyssynchrony [9]. Myocardial scar tissue is not excitable and reduces the extent of the LV myocardium which is responding to pacing stimulus [10]. Cardiac pacing in the area of myocardial scar is associated not only with the suboptimal response to CRT but also with increased risk of cardiovascular death or hospitalization for HF [11].

In the nuclear imaging study, using technecium-99 m tetrofosmin single-photon emission computed tomography (SPECT), an extent and location of the myocardial scar was determined in 51 patients with ischemic HF. Myocardial segments with tracer uptake of more than $75 \%$ were labeled as viable, segments with 50-75\% of maximal tracer activity were considered to contain scar of small extent (non-transmural) and segments with less than $50 \%$ tracer uptake were considered to contain excessive scar (transmural infarction). Patients with transmural scar ( $\mathrm{n}=15,29 \%$ of patients) did not show clinical improvement, reverse remodeling of $\mathrm{LV}$ or increase in $\mathrm{LV}$ function after 6 months follow-up [12].

In another study of myocardial perfusion imaging, using the method of SPECT, the average scar density in the segments in the vicinity of LV lead was lower in responders in comparison to nonresponders [13].

Different results were reported by Riedlbauchova et al. [14]. They assessed myocardial viability in CRT recipients with ischemic HF using a method of positron emission tomography. Simultaneous evaluation of dipyridamole-induced perfusion defect and fluorodeoxyglucose uptake allowed differenciation between viable (hibernating) myocardium and non-viable myocardium (scar). Clinical benefits were observed not only in patients with $\mathrm{LV}$ electrode localized in viable myocardium but also, in lesser extent, in patients with LV electrode positioned in the scar area. These benefits include LV remodeling in patients paced in the area of non-transmural scar and improvement of NYHA class in patients paced in the area of transmural scar.

Areas of myocardial fibrosis can be detected also by non-contact electroanatomical mapping as areas of slow conduction. Location of the LV electrode tip outside of slow conduction areas was associated with the significant hemodynamic improvement in patients with $\mathrm{HF}$ of ischemic and non-ischemic etiology undergoing CRT [15].

Myocardial contractility is a specific marker of myocardium viability. Myocardial deformation imaging can be used to determine myocardial contractility. Peak segmental myocardial strain is related to segmental viability [16]. Becker et al. [17] examined the impact of preserved viability in the area of LV lead on the reverse remodeling and functional status in 65 patients with CRT. Etiology of $\mathrm{HF}$ was ischemic in 46 patients and non-ischemic in 19 patients. They considered the LV segment viable when a peak circumferential strain assessed by speckle tracking imaging exceeded $11.1 \%$. This value was reported to detect transmural MI defined by magnetic resonance imaging with the sensitivity and specificity of about $70 \%$ [16]. Patients with the LV lead placed in a viable segment have greater increase in LVEF and decline in LVEDV than patients with LV electrode in non-viable segment. This difference was more pronounced in patients with ischemic HF than in patients with non-ischemic HF [17].

Longitudinal and circumferential strain rate during low dose dobutamine stress test determined by speckle tracking echocardiography can be used as a measure of myocardial viability and is able to predict functional recovery of the myocardial segment after revascularization [18].

Ran et al. [19] used adenosine as a different method of pharmacological stress testing during echocardiographic examination. Myocardial strain was calculated by the method of speckle tracking echocardiography. Myocardial viability was determined in all myocardial segments using radionuclide imaging of myocardial perfusion and myocardial metabolism. An increase in strain rate identified viable myocardial segments, while non-viable segments exhibited no increase in strain rate [19].

Results of our study are the same as results published by Ypenburg et al. [8]. They used a similar method for the determination of regional contractile reserve and reported an increase in peak strain in the LV lead region during low dose dobutamine stress test $(10 \mu \mathrm{g} / \mathrm{kg} / \mathrm{min})$ in responders to CRT. In non-responders, regional contractile reserve was not present (no increase in strain was observed in the LV lead area).

In our study, we used the higher dose of dobutamine infusion $(20 \mu \mathrm{g} / \mathrm{min})$ than in the previously mentioned study. Despite the more intensive betaadrenergic stimulation, we were not able to show an increase in myocardial contractility in LV lead area in non-responders to CRT. Our study confirms that myocardial viability in the paced area of LV is an important predictor of the response to CRT (AUC 0.852, $\mathrm{p}<0.0001$ ).

Loss of contractile tissue in the vicinity of the LV stimulation electrode due to fibrosis and scar 
reduces the overall contractile force of the LV and alters appropriate sequence of $\mathrm{LV}$ contraction. In this way, it impedes the beneficial effect of CRT on the electrical and mechanical dyssynchrony in the failing heart.

\section{Limitations of the study}

The small amount of patients will require confirmation of the results on the larger study population. The follow-up period of 6 months is relatively short, the long-term effect of CRT needs to be assessed during longer follow-up period. In the present study, only radial myocardial strain was evaluated, determination of longitudinal and circumferential strain during dobutamine stress test could bring additional information.

\section{Conclusions}

The main result of the current study is that regional contractile reserve assessed by strain echocardiography during dobutamine infusion predicts the reverse remodeling of the $\mathrm{LV}$ and response to CRT.

\section{Acknowledgements}

This work was supported by the project "Centre for Excellent Research of Atherosclerosis and its Complications - Myocardial Infarction and Stroke”, Operational Program of Research and Development financed by European Fund for Regional Development.

\section{Conflict of interest: None declared}

\section{References}

1. Dickstein K, Vardas PE, Auricchio A et al. 2010 focused update of ESC Guidelines on device therapy in heart failure: An update of the 2008 ESC Guidelines for the diagnosis and treatment of acute and chronic heart failure and the 2007 ESC Guidelines for cardiac and resynchronization therapy. Developed with the special contribution of the Heart Failure Association and the European Heart Rhythm Association. Eur J Heart Fail, 2010; 12: 1143-1153.

2. Cowburn PJ, Leclercq C. How to improve outcomes with cardiac resynchronisation therapy: Importance of lead positioning. Heart Fail Rev, 2012; 17: 781-789.

3. Singh JP, Klein HU, Huang DT et al. Left ventricular lead position and clinical outcome in the multicenter automatic defibrillator implantation trial-cardiac resynchronization therapy (MADIT-CRT) trial. Circulation, 2011; 123: 1159-1166.
4. Thebault C, Donal E, Meunier C et al. Sites of left and right ventricular lead implantation and response to cardiac resynchronization therapy observations from the REVERSE trial. Eur Heart J, 2012; 33: 2662-2671.

5. Mullens W, Grimm RA, Verga $\mathrm{T}$ et al. Insights from a cardiac resynchronization optimization clinic as part of a heart failure disease management program. J Am Coll Cardiol, 2009; 53: 765-773.

6. Leclercq C, Faris O, Tunin R et al. Systolic improvement and mechanical resynchronization does not require electrical synchrony in the dilated failing heart with left bundle-branch block. Circulation, 2002; 106: 1760-1763.

7. Ramahi TM, Longo MD, Cadariu AR et al. Dobutamine-induced augmentation of left ventricular ejection fraction predicts survival of heart failure patients with severe non-ischaemic cardiomyopathy. Eur Heart J, 2001; 22: 849-856.

8. Ypenburg C, Sieders A, Bleeker GB et al. Myocardial contractile reserve predicts improvement in left ventricular function after cardiac resynchronization therapy. Am Heart J, 2007; 154: 1160-1165.

9. Leyva F. Cardiac resynchronization therapy guided by cardiovascular magnetic resonance. J Cardiovasc Magn Reson, 2010; 12: 64.

10. Breithardt OA, Stellbrink C, Kramer AP et al. Echocardiographic quantification of left ventricular asynchrony predicts an acute hemodynamic benefit of cardiac resynchronization therapy. J Am Coll Cardiol, 2002; 40: 536-545.

11. Chalil S, Stegemann B, Muhyaldeen SA et al. Effect of posterolateral left ventricular scar on mortality and morbidity following cardiac resynchronization therapy. Pacing Clin Electrophysiol 2007; 30: 1201-1209.

12. Ypenburg C, Schalij MJ, Bleeker GB et al. Impact of viability and scar tissue on response to cardiac resynchronization therapy in ischaemic heart failure patients. Eur Heart J, 2007; 28: 33-41.

13. Adelstein EC, Saba S. Scar burden by myocardial perfusion imaging predicts echocardiographic response to cardiac resynchronization therapy in ischemic cardiomyopathy. Am Heart J, 2007; 153: 105-112.

14. Riedlbauchova L, Brunken R, Jaber WA et al. The impact of myocardial viability on the clinical outcome of cardiac resynchronization therapy. J Cardiovasc Electrophysiol, 2009; 20: 50-57.

15. Lambiase PD, Rinaldi A, Hauck J et al. Non-contact left ventricular endocardial mapping in cardiac resynchronisation therapy. Heart, 2004; 90: 44-51.

16. Becker M, Hoffmann R, Kuhl HP et al. Analysis of myocardial deformation based on ultrasonic pixel tracking to determine transmurality in chronic myocardial infarction. Eur Heart J, 2006; 27: 2560-2566.

17. Becker M, Zwicker C, Kaminski M et al. Dependency of cardiac resynchronization therapy on myocardial viability at the $\mathrm{LV}$ lead position. J Am Coll Cardiol Cardiovasc Imaging, 2011; 4: 366-374.

18. Bansal M, Jeffriess L, Leano R, Mundy J, Marwick TH. Assessment of myocardial viability at dobutamine echocardiography by deformation analysis using tissue velocity and speckle-tracking. J Am Coll Cardiol Cardiovasc Imaging, 2010; 3: 121-131.

19. Ran H, Zhang PY, Fang LL et al. Clinic value of two-dimensional speckle tracking combined with adenosine stress echocardiography for assessment of myocardial viability. Echocardiography, 2012; 29: 688-694. 